\title{
BMJ Open Scoping review assessing the evidence used to support the adoption of mobile health (mHealth) technologies for the education and training of community health workers (CHWs) in low-income and middle-income countries
}

\author{
Niall Winters, ${ }^{1}$ Laurenz Langer, ${ }^{2}$ Anne Geniets ${ }^{1}$
}

To cite: Winters N, Langer L, Geniets A. Scoping review assessing the evidence used to support the adoption of mobile health (mHealth) technologies for the education and training of community health workers (CHWs) in low-income and middleincome countries. BMJ Open 2018;8:e019827. doi:10.1136/ bmjopen-2017-019827

- Prepublication history and additional material for this paper are available online. To view these files, please visit the journal online (http://dx.doi org/10.1136/bmjopen-2017019827).

Received 28 September 2017 Revised 3 May 2018 Accepted 13 June 2018

Check for updates

(C) Author(s) (or their employer(s)) 2018. Re-use permitted under CC BY-NC. No commercial re-use. See rights and permissions. Published by BMJ.

${ }^{1}$ Department of Education, University of Oxford, Oxford, UK ${ }^{2}$ University of Johannesburg, Johannesburg, South Africa

Correspondence to Dr Niall Winters; niall.winters@education.ox. ac.uk

\section{ABSTRACT}

Objectives Undertake a systematic scoping review to determine how a research evidence base, in the form of existing systematic reviews in the field of mobile health (mHealth), constitutes education and training for community health workers (CHWs) who use mobile technologies in everyday work. The review was informed by the following research questions: does educational theory inform the design of the education and training component of mHealth interventions? How is education and training with mobile technology by CHWs in lowincome and middle-income countries categorised by existing systematic reviews? What is the basis for this categorisation?

Setting The review explored the literature from 2000 to 2017 to investigate how mHealth interventions have been positioned within the available evidence base in relation to their use of formal theories of learning. Results The scoping review found 24 primary studies that were categorised by 16 systematic reviews as supporting CHWs' education and training using mobile technologies. However, when formal theories of learning from educational research were used to recategorise these 24 primary studies, only four could be coded as such. This identifies a problem with how CHWs' education and training using mobile technologies is understood and categorised within the existing evidence base. This is because there is no agreed on, theoretically informed understanding of what counts as learning.

Conclusion The claims made by mHealth researchers and practitioners regarding the learning benefits of mobile technology are not based on research results that are underpinned by formal theories of learning. mHealth suffers from a reductionist view of learning that underestimates the complexities of the relationship between pedagogy and technology. This has resulted in miscategorisations of what constitutes CHWs' education and training within the existing evidence base. This can be overcome by informed collaboration between the health and education communities.
Strengths and limitations of this study

- The study applied an innovative three-step scoping review methodology to unpack the evidence on mobile technology's contribution to the education and training of CHWs.

- In-depth primary analysis determined if theories of learning were used to conceptualise and categorise education and training in mobile health (mHealth).

- The study details if these theories were used to design and implement the education and training component of mHealth interventions for CHWs.

- The in-depth primary analysis of theories of learning is limited to programmatic information reported in the identified primary studies.

- The review is limited to papers included in systematic reviews published in English between 2000 and 2017.

\section{INTRODUCTION}

The popularity of mobile phones in low-income and middle-income countries (LMICs) has motivated their use in healthcare, particularly as a tool to support primary healthcare outreach by community health workers (CHWs) to those with little or no access to healthcare. CHWs usually receive limited but focused training on key health priorities in LMICs, and they play a vital role in supporting communities to better engage with the formal health system. While the precise scope of their role differs across LMICs (Oliver et al discuss their role in Kenya), they have become a vital part of strategies to address weaknesses in health systems. Mobile technology is increasingly viewed as essential to the work of CHWs.

The field of mobile health (mHealth) investigates the role mobile technologies can play in healthcare. mHealth has many 
functionalities, ${ }^{23}$ one of which is to provide education and training for CHWs. Delivering individual access to educational material is the primary means of achieving this, ${ }^{4}$ particularly in contexts where face-to-face training is limited. Yet, such information dissemination models of education are well known to miss the wider social and cultural aspects of learning inherent to healthcare practice, ${ }^{5}$ and more relevant educational theories, including inquiry learning, experiential learning and situated learning, are used in other areas of healthcare practice. ${ }^{6-8}$

Educational researchers have built on these foundational theories to develop concepts of workplace-based learning and mobile learning, ${ }^{9} 10$ which are designed to support learners to produce new knowledge using technology while working. However, it is unclear if or how workplace-based learning and mobile learning research has been incorporated into mHealth platforms. Preliminary indicators suggest that almost all 'education theory' is ignored. For example, in Labrique et $a l \mathrm{~s}^{11}$ widely regarded mHealth framework, none of the example interventions in the category "provider training and education' (p. 164) are informed by formal theories of workplace-based or mobile learning. Other categories, such as 'electronic decision-support' could be considered workplace-based learning. The problem is further complicated by the fact that in two systematic reviews of mHealth interventions, ${ }^{3} 12$ the same underlying mechanisms of information dissemination and increased communication are applied to two very different challenges: (A) patient education for behaviour change and (B) CHWs' continuous professional development. Yet, from an educational perspective, it is challenging to equate mHealth interventions that provide health-related information with interventions trying to change CHW's practice and support professional development; the underlying pedagogical mechanisms required for both types of interventions differ significantly in nature and scale.

Consequently, there is a pressing need to understand: (1) if and how educational theories are being incorporated into mHealth platforms currently? (2) How the adoption of a novel educational lens can inform the future development of mHealth technology for use by CHWs?

While multiple reviews of mHealth in LMICs have recently been published, this systematic scoping review is the first to combine theories of workplace-based learning and mobile learning and apply them to mHealth research on education and training for CHWs in LMICs. The focus of the review is not on measurable endpoints of education and training, but rather on how the educational components have been conceptualised within existing mHealth research.

\section{METHODS}

\section{Review approach}

We conducted a systematic scoping review of the research evidence on the use of mobile technologies to facilitate
CHWs' education and training in LMICs. A scoping review is defined ${ }^{13}$ as 'a form of knowledge synthesis that addresses an exploratory research question aimed at mapping key concepts, types of evidence, and gaps in research related to a defined area or field by systematically searching, selecting, and synthesising existing knowledge' (p. 1292). Scoping reviews are part of the family of research synthesis methods but, compared with systematic reviews, address broader research questions. They aim to provide an overview and organisation of existing knowledge rather than a narrow synthesis of a predefined research question. ${ }^{14}{ }^{15}$ Usually, this different synthesis approach is conducted over a shorter timeframe than systematic reviews, using more targeted search terms and focuses less on the critical appraisal of the included evidence.

A scoping review approach was chosen for this study because we wanted to explore how existing literature has conceptualised and operationalised the use of mobile technologies to support CHWs' learning practices. The focus is on the diversity of understandings and definitions of CHWs' education and training in the existing literature and what patterns and gaps might emerge from a systematic analysis of this body of knowledge. ${ }^{i}$ In order to capture the conceptualisation and positioning of mHealth interventions that have an education or training component, our scoping review targeted existing systematic reviews of mHealth interventions rather than primary studies as a first level of analysis. Unlike primary studies, these reviews require an explicit conceptual frameworkincluding Labrique's framework-in order to group mHealth interventions for analysis. Consequently, we can derive the positioning and categorisation of different mHealth interventions with respect to their support for CHWs' education and training from these systematic reviews.

Our scoping review followed explicit and transparent research steps to explore the research evidence on mHealth and CHWs' education and training. A review protocol was not published, and the study was not registered with PROSPERO, as these mechanisms are not applied to scoping reviews. ${ }^{13} 14$

We followed a novel three-step approach in our scoping review that combined secondary research methods with a primary reanalysis of the included studies. In the first step, existing systematic reviews investigating CHWs' education and training when using mobile technologies were sought. As outlined above, this novel approach was necessary to allow us to investigate how different mHealth interventions were categorised in relation to education and training within the evidence base. In the second step, we then extracted the primary studies included in these reviews in order to provide a descriptive account of the

${ }^{\mathrm{i}}$ We were not concerned with whether mobile technologies are effective in increasing learning outcomes or how CHWs perceive the use of mobile technologies. These types of research questions lend themselves to full systematic reviews. 
included mHealth interventions and the wider characteristics of the evidence base. In the third step, we conducted a primary reanalysis of the included mHealth interventions, which were recoded with respect to their education or training component. That is, we used two coding frameworks inspired by different theories of learning: workplace-based learning and mobile learning. ${ }^{9} 10$ These two theories were selected because they are both well developed, proven and have been applied in multiple projects in the education literature. Each builds on over a decade of research and draws together key conceptual points into practically applicable frameworks.

The chosen coding frameworks were then applied to the primary studies included in the systematic review. As a result, we obtained two different set of results on how mHealth interventions were categorised regarding their support for CHWs' education and training: (1) the categorisation of interventions in the systematic reviews themselves and (2) our recategorisation of the same interventions using explicit learning from educational research. These two sets of categorisations allowed us to juxtapose the prevailing positioning and understanding of education and training in mHealth with a more pedagogically grounded understanding. A more traditional review approach, without this reanalysis of primary studies, would not have allowed us to juxtapose these different understandings. The same applies had we followed a systematic review approach that only included primary studies and not the existing reviews themselves. We elaborate on the methods employed in each step below.

\section{Step 1: review of existing systematic reviews \\ Search methods}

We designed an exhaustive and sensitive search strategy to identify all relevant reviews of mHealth interventions that included CHWs' education and training facilitated by mobile technologies in LMICs. The search strategy was deliberately designed to be overinclusive. Search terms were formulated to identify any mHealth review covering LMICs, and we manually filtered down the reviews relevant to CHWs' education and training. Likewise, despite being focused on CHWs in our review, our search strategy did not specify terms related to CHWs. Both decisions ensured that no relevant reviews were excluded during the search. The full search terms therefore only included key words for the concepts 'mHealth', 'systematic review' and 'LMICs'. Concepts were combined using the AND boolean operator to develop a master search string (online supplementary material 1 ).

The full search string was then applied to a range of academic databases in the health and social sciences: CINAHL, PubMed; MEDLINE, PsychInfo, ERIC, Education Full-text and ISI Web of Science. Database searches covered the period 2000-2017. The year 2000 as a cut-off date was chosen as mobile technologies did not see widespread application to support healthcare in LMICs before then. In addition, we also searched the grey literature for reviews relevant for inclusion. Grey literature sources included Google and Google Scholar searches as well as specialised systematic review databases, that is, Cochrane Library of Systematic Reviews, Campbell Library and the 3ie database of international development reviews. Lastly, reference lists of included reviews were used as an additional source for snowball searching for additional reviews. A full record of the conducted search is provided in online supplementary material 2.

\section{Inclusion criteria}

We formulated explicit inclusion criteria that determined what reviews were eligible for inclusion in our scoping review. Conceptually, this referred to existing systematic reviews of mHealth interventions that support CHWs' education and training in LMICs. To operationalise this into transparent inclusion criteria, the following definitions were applied.

\section{Population}

CHWs were defined broadly in line with the WHO's 2007 definition of lay health workers as applied in Lewin et $a l^{16}$ :

Community health workers should be members of the communities where they work, should be selected by the communities, should be answerable to the communities for their activities, should be supported by the health system but not necessarily a part of its organization, and have shorter training than professional workers. (p. 3)

This definition allows for different types of healthcare workers to be classified as CHWs in different contexts. Reviews were included as long as they covered mHealth interventions applied by or for CHWs regardless of whether CHWs were the main focus of the review. LMICs were defined using the World Bank classification of economies. ${ }^{17}$ To be included, reviews had to focus on LMICs and for reviews that had no regional scope, at least $50 \%$ of the included studies had to be from LMICs in order for the review to be featured in our scoping review.

\section{Intervention}

Reviews had to include mHealth interventions that used mobile technology to facilitate CHWs' education and training. This excludes reviews that focus on fixed ICT infrastructure such as desktop PCs and fixed diagnostic ICTs only. Education and training was defined broadly, and we followed the reviews' positioning of interventions as to how they facilitated learning. In addition, we included the following categories used in reviews based on Labrique $e t$ $a l$ s framework to ensure no relevant interventions were missed: decision support, provider-provider communication, provider work planning and scheduling, data collection and reporting. A systematic review covering any of the above categories was thus included in our scoping review. This was because each of these categories could potentially be framed as supporting the CHWs' education and training using conceptualisations of workplace-based 
learning and mobile learning. For example, improved communication between CHWs could support collaboration and social learning. Likewise, following explicit decision-making algorithms could lead to the learning and acquisition of new and improved practices by CHWs. Both 'provider work planning and scheduling' and 'data collection and reporting' can offer CHWs with opportunities for reflective practice, for example, by providing insights into the relationship between data capture and decision making. Reviewing cohort data could offer supervisors the opportunity to support peer learning. Again, we aimed to be overinclusive at this stage so as not to miss any relevant reviews.

\section{Research design}

To be included, studies had to qualify as a 'systematic review', which was defined broadly for this scoping review. Any type of research synthesis was included as long as a structured and transparent review approach was applied. Indicators of a structured review approach referred to: reporting of (1) a systematic search; (2) predefined inclusion criteria; and (3) a stated method of synthesis. Indicators of a transparent review approach referred to: reporting of (1) numbers of searched and included studies; (2) a summary table of included studies; and (3) a discussion of the strengths of the evidence in the synthesis.

\section{Outcomes}

No studies were excluded on the basis of measured outcomes or applied outcome measures because intervention effectiveness was not of concern in this scoping review.

\section{Screening and coding of reviews}

Two reviewers screened all search hits for potentially relevant systematic reviews at title and abstract. Full-texts of potentially relevant reviews were then sought and screened again against our inclusion criteria. A subset of $10 \%$ of the citations eligible of full-text screening were double-screened to assess inter-reviewer reliability. Disagreements between reviewers were resolved by joint discussion with a third reviewer acting as an arbitrator. Following the screening, included systematic reviews were then coded for two high-level characteristics: (1) applied framework to categorise interventions and (2) included mHealth interventions.

\section{Critical appraisal}

As this is a scoping review, no critical appraisal of either included reviews or primary studies was conducted.

\section{Step 2: extraction of primary studies from the included reviews}

Identification of primary studies

Having identified eligible reviews, we then extracted the primary studies included in each review for further analysis. That is, the included systematic reviews served as the data source for primary studies. We only searched the relevant systematic reviews under the intervention categories that could potentially relate to education and training. Extracting only primary studies that existing systematic reviews had coded and categorised as related to education and training allows us to unpack and examine this positioning.

We did not conduct an independent scientific search for relevant primary studies in addition to the search for systematic reviews. Including primary studies that were not found in existing systematic reviews would not have revealed any new information regarding how the primary studies were categorised. As a result, primary studies of mHealth interventions and CHWs that were not included in any of the systematic reviews were excluded from our scoping review. In practice, this refers mainly to primary studies published after the included systematic reviews were conducted. (Searches for the two most up-to-date systematic reviews ${ }^{18} 19$ included in our scoping review were completed in December 2015.)

\section{Inclusion criteria for primary studies}

In terms of population, intervention and outcome, the inclusion criteria of the primary studies were identical to the criteria for systematic reviews. In terms of study design, however, primary studies could be of any empirical research design that investigated an applied mHealth intervention. This included both quantitative and qualitative research designs but excluded designs that assessed interventions in a lab setting and/or assessed perceptions and feasibility of a future intervention implementation. ${ }^{2021}$

\section{Screening and coding of primary studies}

All primary studies allocated to the eligible intervention categories explained above were screened at full text by two reviewers. The same quality assurance processes as used for the screening of the systematic reviews were implemented. We designed an explicit coding tool to capture key characteristics related to the type of CHWs, the type of mHealth intervention and technology applied, the context in which it was applied, as well as the educational event or process facilitated by the technology.

\section{Step 3: primary analysis of study's categorisation as supporting the education and training of CHWs}

Two well-established coding frameworks from educational research feature the key pedagogical attributes of workplace-based and mobile learning: Eraut and Hirsh ${ }^{9}$ for workplace-based learning and Kearney et $a l^{10}$ for mobile learning. Applying these two frameworks as our coding tool allowed us to recode the primary studies in order to investigate whether their claim to facilitate CHWs' education and training did hold true from a pedagogical perspective. In this last step, we thus can compare the outcomes of this pedagogically informed coding tool with the reported codes in the reviews. Again, two independent reviewers applied the coding tool with a third reviewer acting as an arbitrator in case of disagreement. 


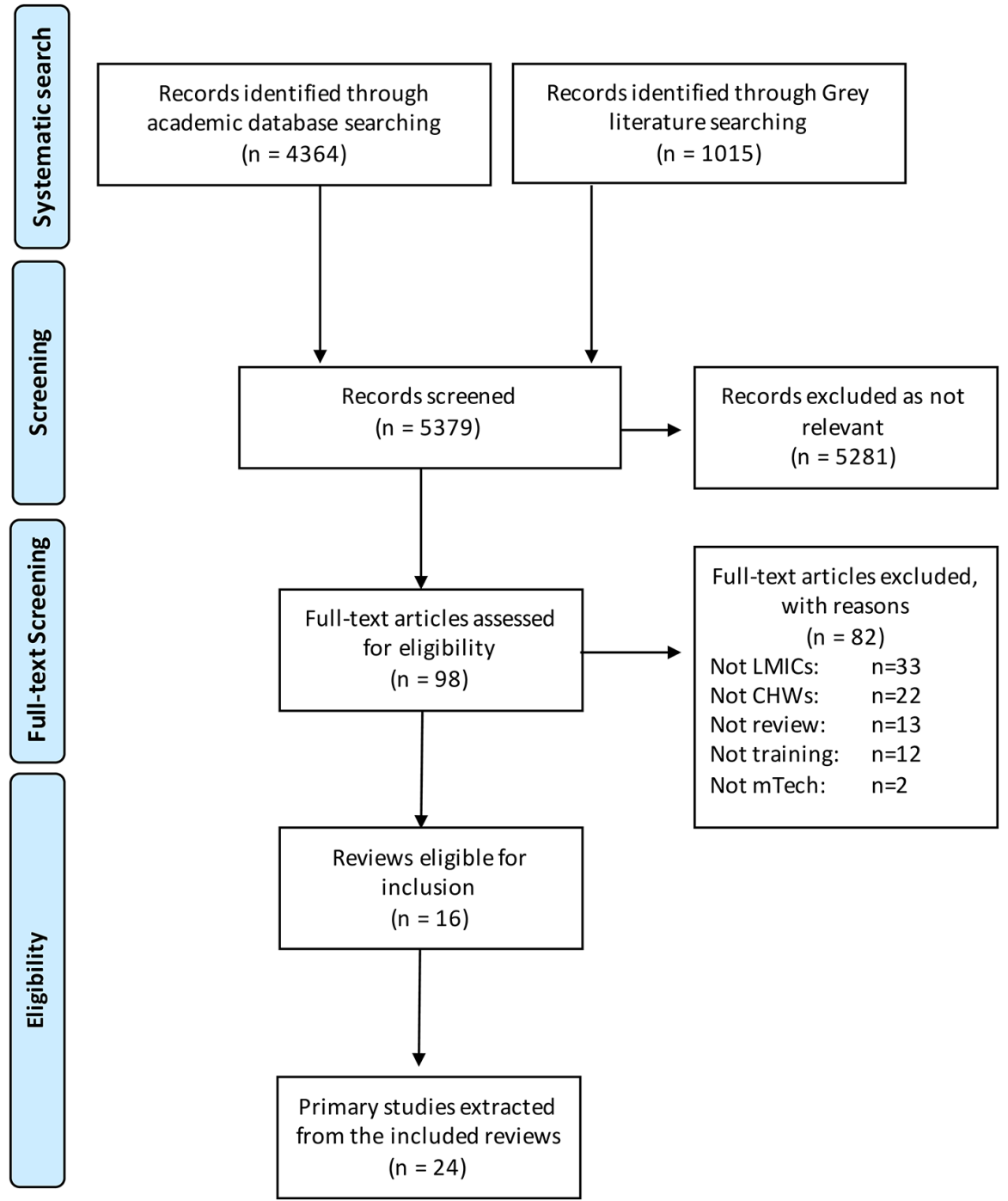

Figure 1 PRISMA flow chart of identification and inclusion of studies. CHWs, community health workers; LMICs, lowincome and middle-income countries; PRISMA, Preferred Reporting Items for Systematic Reviews and Meta-Analyses.

\section{Review limitations}

There are three key limitations to this review. First, only English-language articles were considered for inclusion. Second, systematic reviews published up to 2017 only cover primary studies published up to 2015. Studies published after this date were not identified by the systematic reviews and by extension are not covered by our scoping review. In general, relying on systematic reviews as an identification strategy entails the risk that our review is subject to a limitation in scope because we can only reproduce the scope of the included systematic reviews in our own review. ${ }^{\text {ii }}$ Third, only a partial range of grey literature was searched, and mHealth conferences were not covered.

\section{Patient and public involvement}

Patients or the public were not involved in this research.

\footnotetext{
${ }^{\mathrm{ii}}$ However, this limitation is mitigated by the large number of identified systematic reviews $(n=16)$, which provide large depth and breadth in the scope of included systematic reviews and thus in our own scoping review.
}

\section{FINDINGS}

\section{Search results}

Searches were run between March and May 2016 and updated in June 2017. They yielded a total of 5379 citations from 12 different sources (figure 1). After screening these citations on title and abstract, the large majority of citations were not relevant $(n=5281)$ - a result of our deliberately overinclusive search strategy. We identified 98 existing reviews that on title and abstract met our inclusion criteria. Full texts of these reviews were then sought, and reviews screened for inclusion in more detail. This in-depth screening excluded a further 82 reviews leaving only 16 reviews that met the predefined inclusion criteria. Reasons for exclusion at full-text screening referred to: reviews not including studies from LMICs $(n=33)$; not including studies that focus on CHWs as a population $(n=22)$; not classified as following a structured and transparent review method $(\mathrm{n}=13)$; not including studies that focus on mobile technologies to facilitate training and learning $(\mathrm{n}=12)$; and not including studies that focus on mobile technologies $(n=2)$. As a result, we were left with 
16 reviews that included research evidence on the application of mobile technologies to facilitate CHWs' education and training in LMICs.

In a second step, we then extracted the primary studies included in the 16 systematic reviews. We only extracted primary studies that were coded in the reviews to fit intervention categories associated with $\mathrm{CHWs}$ ' education and training. As explained above, this referred to: provider training and education, decision support, provider-provider communication, provider work planning and scheduling, and data collection and reporting. Controlling for duplicates, we identified 24 studies that were included in the systematic reviews. Online supplementary material 3 provides a list of all systematic reviews and primary studies that were included in our scoping review.

\section{Description of mHealth interventions: how did they facilitate CHWs' education and training?}

We extracted descriptive information from all 24 included primary studies using a structured coding tool. A summary table of the extracted data per study is presented in online supplementary material 4 . Of the 24 primary studies extracted from the 16 systematic reviews, three were undertaken in Kenya, three in Malawi, three in Tanzania, two in Ghana, two in Rwanda and two in South Africa. One study was undertaken in: Bangladesh, Ethiopia, Guatemala, Nigeria, Liberia, Uganda, India and Pakistan, respectively. One study was conducted in multiple countries (Mexico and Guatemala). Seventeen studies were undertaken in a rural setting, one in both urban and rural, one periurban and four urban, with one setting undetermined. SMS was used in eight of the studies (including RapidSMS and FrontlineSMS), CommCare in five studies, MoTECH in one, Java Applets in three, customised designs in three studies and standard voice calls in two studies. One tool was undetermined, and one used a Palm Pilot PDA.

Intervention participants were described as CHWs in 13 studies, while 2 studies referred to traditional birth attendants. In nine studies, different terms were used to describe healthcare workers fitting the above definition of CHWs. Examples of these include: village elders, community health volunteer, health surveillance assistant and accredited social health activist. The number of CHWs involved in the mHealth interventions ranged from 5 to 638 with a median of 75 . Only seven studies reached more than 100 CHWs. In all but one study, ${ }^{22}$ the CHWs involved in the mHealth intervention were synonymous with the research sample.

The 24 studies included in the 16 systematic reviews reported a range of mHealth interventions that were positioned to facilitate CHWs' education and training. Of the 24 extracted primary studies, a majority were grouped by the systematic reviews to provide direct training and education to CHWs ( $\mathrm{n}=16$ ) (figure 2). This could refer, for example, to using mobiles to facilitate continued professional development. A similar number of studies used mobile devices to enhance the communication between CHWs as well as with their supervisors $(n=14)$. For instance, through use of SMS feedback and rapid response services in order to enhance CHWs' access to information and support learning. Other common intervention categories referred to the application of mobiles to train CHWs to collect and manage medical data $(n=13)$, the use of technology-supported decision-making tools $(n=11)$ and the facilitation of supervision of CHWs $(n=8)$.

\section{Methodological approaches}

A variety of methodological approaches with a range of research methods and designs were used: case study $(\mathrm{n}=7)$, pilot study $(\mathrm{n}=6)$, mixed methods $(\mathrm{n}=4)$ and quasiexperimental designs $(n=4)$. There were only two RCTs and one technical evaluation, and one study where the methods used could not be determined.

\section{Discrepancies in categorisation}

The same studies reported by different reviews were not consistently categorised in their relation to CHWs' education and training. For example, a study that reported on mobile phone text message reminders to support Kenyan health workers' adherence to malaria treatment guidelines $^{23}$ was included in five reviews but was alternatively categorised as a decision support tool, a monitoring and compliance device or as a training and education intervention (online supplementary material 5 ). This pattern characterises the entire sample, where there is a large overlap between the primary studies included in the systematic reviews, but little overlap in their allocation to intervention categories. On average, each primary study is allocated to three different intervention categories across different or within reviews. Of the primary studies allocated to multiple categories $(n=14)$, only four studies are consistently allocated to the same intervention category across reviews (see online supplementary material 5 , column 1). As a result, there seems to be little agreement between reviews regarding what type of interventions can directly facilitate CHWs' education and training and how such learning can be defined.

This is not surprising given that the challenges of categorisation are well known. ${ }^{24}$ However, given the large variance in the allocation of interventions, there is a need for mHealth researchers to develop a clearer understanding of what counts as education and training for CHWs. To overcome the seemingly ad hoc manner of categorisation, we used educational research to develop a refined coding tool (see online supplementary materials 5 and 6) This tool is based on pedagogical frameworks for workplace and mobile learning ${ }^{910}$ and is applied to assess the exact nature of education and training that was supported by the mHealth interventions.

\section{Recoding of mHealth interventions using educational frameworks}

The results of our reanalysis of the included primary studies and whether the reported mHealth interventions 


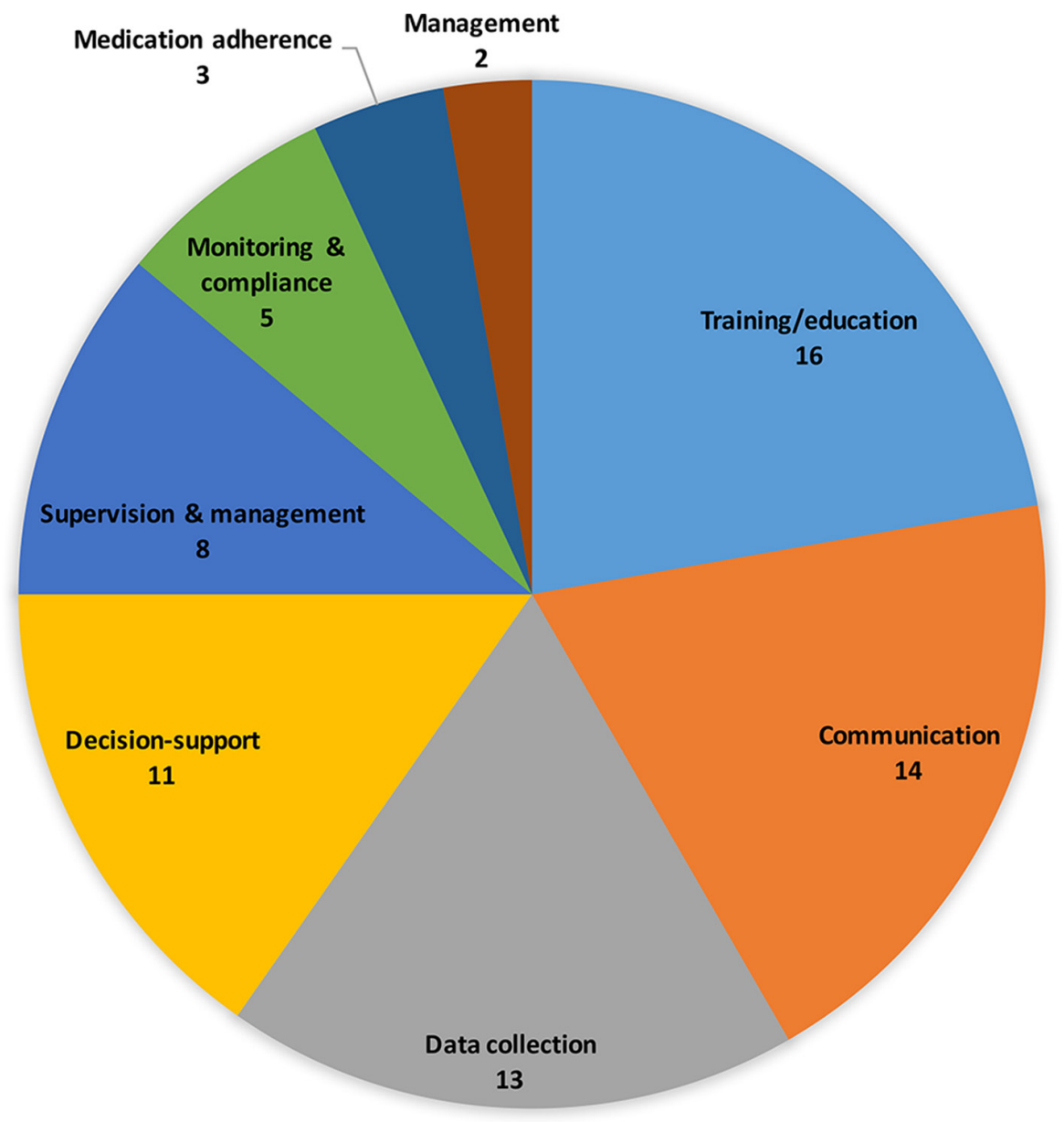

Figure 2 Overview of mHealth intervention categories taken directly from the 16 included systematic reviews. The primary studies were often characterised differently by different systematic reviews. mHealth, mobile health.

could indeed be positioned to facilitate CHWs' education and training through the use of mobiles are presented in online supplementary material 5 .

In online supplementary material 5 , columns 2 and 3 show the findings of our recoding of whether the interventions can be classified as workplace learning (column 2) or mobile learning (column 3). The key criterion to determine if an intervention supports practice-based mobile learning was that at least one aspect of workplace-based learning and one aspect of mobile learning were addressed (see online supplementary material 6 for the coding tool). From recoding the primary studies using the educational frameworks, we find that only four mHealth interventions ${ }^{2325-27}$ could be positioned as facilitating CHWs' education and training through the use of mobile technology. That is, of the 24 studies that are allocated in the systematic reviews to categories associated with a potential educational use of technology, the allocation of 20 studies cannot be confirmed from a pedagogic perspective. The only four studies where the allocation can be confirmed are highlighted in green in online supplementary material 5 .

\section{DISCUSSION}

Of the four studies that remained after recoding, ${ }^{23}$ 25-27 all exhibited elements of workplace-based and mobile learning (see online supplementary material 6). However, the ways in which these elements were implemented was weak from an educational research perspective. The need to produce evidence on how mobile technology can support reflective and interactive forms of CHWs' education and training, particularly coaching, supervision and mentoring, remain critically neglected overall. mHealth interventions are not building effectively enough on previous global health research, ${ }^{28}$ which has evidenced how good-quality supervision 'is one the key approaches to improving the quality of health care' (p. 3). This is particularly true when it is backed up by regular support and feedback. ${ }^{29-34}$

Instead, priority seemed to be given to easily scalable basic technologies that use an information dissemination model of learning to ensure CHW adherence to standardised practice (eg, simplified guidelines on protocols sent via text messages). Learner agency was not a core priority. In three out of the four studies, ${ }^{2325} 26$ agency was trumped by the need for CHWs to be held accountable to managerial oversight. Simplistic approaches, which focus on measuring the volume of information that CHWs are exposed to as a proxy for education and training, have their limitations. It is unclear how they can empower CHWs, and there is a danger that the drive for improved 
efficiency through mobile technology could have the adverse effect of deprofessionalisation, ${ }^{35}$ potentially leading to a weakening of community-based health structures in the longer term. Instead, mHealth training interventions need to be seen as part of a wider learning health systems approach ${ }^{36}$ to support the training of CHWs and as such cannot be considered in isolation.

Decision-support tools offer a means by which to generate insights into CHW practice and could be used as a medium to improve learning. However, the ways in which this could be implemented were not explored in detail. Svoronos $e t a t^{26}$ chose to focus on the details of system implementation. While in Blaschke et $a l^{25} \mathrm{CHWs}$ 'stated that they felt empowered' (p. 24) by the automatic provision of a patient's weight for height calculation, but how this impacted on changes in practice through improved learning was not provided.

Instead of focusing on the developmental needs of CHWs, mHealth interventions in the main concentrate on providing CHWs with tools to support activities for which they may not know the wider significance due to lack of training. Jones $e t a l^{37}$ (the qualitative evaluation of the Zurovac et a $l^{23}$ RCT) are open in the weaknesses of this aspect of their work: 'it was clear that many of the participants believed that the type of training they received did not provide them with an adequate understanding of the importance of the new knowledge, or of the positive outcomes that a change in practice could bring' (p. 4). They also noted that 'few participants mentioned the messages in terms of "support supervision", rather that they were made to feel somewhat guilty for not employing proper practice'. ${ }^{37}$ This is not surprising as using mobile technology for such a nuanced task as supportive supervision is challenging. Our previous work has demonstrated how CHWs and their supervisors used mobile messaging platforms (eg, Whatsapp) to engage in virtual one-to-one, group and peer-to-peer forms of supportive supervision. ${ }^{38}$ Additional research is needed to investigate how technology can be embedded within successful supportive supervisory systems.

The one study that matched most closely what is known about mobile and workplace learning was MartínezFernández et al..$^{28}$ In this study, mobile phones were used by $125 \mathrm{CHWs}$ 'to make consultations regarding issues about which they are unsure; send full epidemiological and clinical information related to the cases they attend; receive continuous training, and perform community health promotion and prevention activities through distance learning sessions' (p. 284). After being given some technical training on phone use and data collection, they were provided with basic initial training in vital signs monitoring and in the identification of signs of distress in children and pregnant women. The CHWs could use their phones for teleconsultations with medical staff (gynaecologists, paediatricians, internists and surgeons). Quarterly face-to-face training was augmented by teletraining. It is very clear that this intervention had a much stronger focus on 'work processes as a by-product

Table 1 Summary of the implications of our key discussion points

\section{Key discussion points}

There is a large overlap between the primary studies included in the systematic reviews but little overlap in their allocation to intervention categories.

As a result, there seems to be little agreement between reviews regarding what type of interventions can directly facilitate CHWs' education and training and how such learning can be defined.

We used educational research to develop a refined coding tool

\section{Implications}

Potential for misclassification. Further research is required to determine more robust classification categories.

There is not a coherent evidence base due to a lack of primary studies explicitly detailing the role of their interventions in learning and training.

The coding tool we used is available in online supplementary material 6. Details of the theories it is based on is available in online supplementary material 7 .

The tool was designed to be refined as the evidence base develops.

The need to produce evidence on how mobile technology can support reflective and interactive forms of CHWs' education and training, particularly coaching, supervision and mentoring, remain critically neglected overall.

Focus on the developmental needs of CHWs to improve their practice.

The review has highlighted the need for more evidence on the precise nature of CHWs' education and training that can be supported by mHealth interventions.
A new interdisciplinary research agenda on training and education in mHealth that builds on existing global health research and moves away from information dissemination model of learning is needed.

Targeted training approaches that use new technologies in innovative ways to promote CHWs' CPD are required. In particular, theories of work-based learning need to be better implemented.

CHWs' decision support tools would have the means to offer insights into CHWs' learning, but studies generally do not explore learning aspects of these tools. At most, learning is seen as a positive by-product. We argue that primary studies should include better documentation of learning practices where applicable.

CHWs, community health workers; CPD, continued professional development; mHealth, mobile health. 
of learning' including 'consultation' and 'embedding on-going training within the intervention design', a fact overlooked by the other interventions. The key findings are summarised in table 1 .

\section{Limitations}

Our scoping review only covers systematic reviews published up to 2017, which means that only primary studies published up to 2015 were included. Our work is open to the biases inherent in relying on existing systematic reviews. However, the scoping review seems well designed to deal with these: first, we included a large number of systematic reviews $(n=16)$, ensuring a wide coverage of primary studies included in these reviews. Second, we further reanalyse the studies included in these reviews to mitigate any quality concerns regarding the included systematic reviews themselves. In targeting secondary literature, we rely on education researchers' interpretation of mobile learning and workplace-based learning in order to unpack patterns in categorisations and conceptualisations. We have made this process transparent through inclusion of our coding tool (see online supplementary material 6) and references. ${ }^{9} 10$ 38-48 Other researchers may take an alternative perspective on this literature.

\section{CONCLUSION}

The findings from this scoping review suggest the mHealth literature is in danger of overclaiming regarding its ability to promote CHWs' education and training within a community work context. Studies claiming to have an educational component to their mHealth intervention were not often informed by educational theory nor was the educational approach taken well documented. The review has highlighted the need for more evidence on the precise nature of CHWs' education and training that can be supported by mHealth interventions. This needs to start with improved categorisation, building on educational frameworks and richer accounts of learning. ${ }^{39}$ The mechanisms for achieving educational outcomes are still unknown, and educational theory should be embedded in the design of an intervention as well as in its evaluation, for which further cross-disciplinary work between global health and education is needed. Appropriate models of technology-enhanced learning ${ }^{40-42}$ and extended use of educational theories will enable the development of much needed robust evidence on the role of technology in supporting CHWs' education and training in mHealth. Achieving this will be challenging, given the complex realities of using mHealth in low-income settings. Nevertheless, we promote ${ }^{49}$ the use of training tools that employ empirically proven equitable pedagogic strategies to maximise learning as a continual process of 'participation' ${ }^{43}$ within a social justice approach to global health. ${ }^{50}$

Acknowledgements We would like to thank Dr Shobhana Nagraj and also the five reviewers for their insightful comments on the paper.
Contributors Activities undertaken by the authors were as follows: establishment of research question/s and development of search strategy: NW and LL. Background framing: NW and AG. Database search and record screening: LL. Extraction of primary studies from the included reviews: LL and NW. Recoding: NW and LL. Discussion and conclusion: all authors.

Funding This research received no specific grant from any funding agency in the public, commercial or not-for-profit sectors.

Competing interests None declared.

Patient consent Not required.

Provenance and peer review Not commissioned; externally peer reviewed.

Data sharing statement All relevant data are within the paper and supporting material.

Open access This is an open access article distributed in accordance with the Creative Commons Attribution Non Commercial (CC BY-NC 4.0) license, which permits others to distribute, remix, adapt, build upon this work non-commercially, and license their derivative works on different terms, provided the original work is properly cited, appropriate credit is given, any changes made indicated, and the use is non-commercial. See: http://creativecommons.org/licenses/by-nc/4.0/.

\section{REFERENCES}

1. Oliver M, Geniets A, Winters N, et al. What do community health workers have to say about their work, and how can this inform improved programme design? A case study with $\mathrm{CHWs}$ within Kenya. Glob Health Action 2015;8:27168.

2. Braun R, Catalani C, Wimbush J, et al. Community health workers and mobile technology: a systematic review of the literature. PLOS One 2013;8:e65772.

3. Hall CS, Fottrell E, Wilkinson S, et al. Assessing the impact of mHealth interventions in low- and middle-income countries--what has been shown to work? Glob Health Action 2014;7:25606.

4. O'Donovan J, Bersin A, O'Donovan C. The effectiveness of mobile health ( $\mathrm{mHealth}$ ) technologies to train healthcare professionals in developing countries: a review of the literature. BMJ Innov 2015;1:33-6.

5. Bleakley A. Broadening conceptions of learning in medical education: the message from teamworking. Med Educ 2006;40:150-7.

6. Sturmberg JP, Farmer L. Educating capable doctors--a portfolio approach. Linking learning and assessment. Med Teach 2009;31:e85-9.

7. Williams PL. Using theories of professional knowledge and reflective practice to influence educational change. Med Teach 1998;20:28-34.

8. Taylor DC, Hamdy H. Adult learning theories: implications for learning and teaching in medical education: AMEE Guide No. 83. Med Teach 2013;35:e1561-72.

9. Eraut M, Hirsh W. The significance of workplace learning for individuals, groups and organisations. Skope Monograph Number;9 http://www.skope.ox.ac.uk/?person=the-significance-of-workplacelearning-for-individuals-groups-and-organisations.

10. Kearney M, Schuck S, Burden K, et al. Viewing mobile learning from a pedagogical perspective. Research in Learning Technology 2012;20:14406.

11. Labrique $A B$, Vasudevan $L$, Kochi $E$, et al. mHealth innovations as health system strengthening tools: 12 common applications and a visual framework. Global Health: Science and Practice 2013;1:160-71.

12. Aranda-Jan CB, Mohutsiwa-Dibe N, Loukanova S. Systematic review on what works, what does not work and why of implementation of mobile health (mHealth) projects in Africa. BMC Public Health 2014;14:188.

13. Colquhoun $\mathrm{HL}$, Levac D, O'Brien KK, et al. Scoping reviews: time for clarity in definition, methods, and reporting. $J$ Clin Epidemiol 2014;67:1291-4.

14. Arksey H, O'Malley L. Scoping studies: towards a methodological framework. Int J Soc Res Methodol 2005;8:19-32.

15. Miake-Lye IM, Hempel S, Shanman R, et al. What is an evidence map? A systematic review of published evidence maps and their definitions, methods, and products. Syst Rev 2016;5:28.

16. Lewin S, Munabi-Babigumira S, Glenton C, et al. Lay health workers in primary and community health care for maternal and child health and the management of infectious diseases. Cochrane Database Syst Rev 2010:CD004015.

17. World Bank. Country classifications. http://blogs.worldbank.org/ opendata/category/tags/news (Accessed September 2017). 
18. Adepoju IO, Albersen BJ, De Brouwere V, et al. mHealth for Clinical Decision-Making in Sub-Saharan Africa: A Scoping Review. JMIR Mhealth Uhealth 2017;5:e38.

19. Tian M, Zhang J, Luo R, et al. mHealth Interventions for Health System Strengthening in China: A Systematic Review. JMIR Mhealth Uhealth 2017;5:e32.

20. Florez-Arango JF, lyengar MS, Dunn K, et al. Performance factors of mobile rich media job aids for community health workers. J Am Med Inform Assoc 2011;18:131-7.

21. lyengar MS, Florez-Arango JF. Decreasing workload among community health workers using interactive, structured, rich-media guidelines on smartphones. Technol Health Care 2013;21:113-23.

22. DeRenzi B, Findlater L, Payne J, et al. Improving community health worker performance through automated SMS. In: Proceedings of the Fifth International Conference on Information and Communication Technologies and Development 2012:25-34.

23. Zurovac D, Sudoi RK, Akhwale WS, et al. The effect of mobile phone text-message reminders on Kenyan health workers' adherence to malaria treatment guidelines: a cluster randomised trial. Lancet 2011;378:795-803

24. Bowker GC, Star SL. Sorting things out: Classification and its consequences. Cambridge: MIT Press, 2000.

25. Blaschke S, Bokenkamp K, Cosmaciuc R, et al. Using mobile phones to improve child nutrition surveillance in Malawi. Brooklyn, NY: UNICEF Malawi, UNICEF Innovations, Mobile Development Solutions, 2009.

26. Svoronos T, Mjungu P, Dhadialla R, et al. CommCare: Automated quality improvement to strengthen community-based health. Weston, Mass.: D-Tree International, 2010.

27. Martínez-Fernández A, Lobos-Medina I, Díaz-Molina CA, et al. TulaSalud: An m-health system for maternal and infant mortality reduction in Guatemala. J Telemed Telecare 2015;21:283-91.

28. Marquez L, Kean L. Making supervision supportive and sustainable: new approaches to old problems. MAQ Working Paper https://www. k4health.org/sites/default/files/maqpaperonsupervision.pdf.

29. Ashwell HE, Freeman P. The clinical competency of community health workers in the eastern highlands province of Papua New Guinea. P N G Med J 1995;38:198-207.

30. Baqui $\mathrm{AH}$, Arifeen $\mathrm{SE}$, Rosen $\mathrm{HE}$, et al. Community-based validation of assessment of newborn illnesses by trained community health workers in Sylhet district of Bangladesh. Trop Med Int Health 2009;14:1448-56.

31. Bhattacharji S, Abraham S, Muliyil J, et al. Evaluating community health worker performance in India. Health Policy Plan 1986;1:232-9.

32. Bhattacharyya K, LeBan K, Winch $\mathrm{P}$, et al. Community health workers: incentives and disincentives: how they affect motivation, retention, and sustainability. Arlington, Virginia: Published by the Basic Support for Institutionalizing Child Survival Project (Basics II) for the United States Agency for International Development, 2001.

33. Laughlin M. The Care Group difference: A guide to mobilizing community-based volunteer health educators. Baltimore, Md, USA: World Relief, 2004
34. Lehmann U, Sanders D. Community health workers: what do we know about them. The state of the evidence on programmes, activities, costs and impact on health outcomes of using community health workers. Geneva: World Health Organization, 2007.

35. Winters N, Oliver M, Langer L. Can mobile health training meet the challenge of 'measuring better'? Comp Educ 2017;53:115-31.

36. Irimu G, Ogero M, Mbevi G, et al. Clinical Information Network Authors Group. Approaching quality improvement at scale: a learning health system approach in Kenya. Arch Dis Child 2018:archdischild-2017-314348.

37. Jones CO, Wasunna B, Sudoi R, et al. "Even if you know everything you can forget": health worker perceptions of mobile phone textmessaging to improve malaria case-management in Kenya. PLoS One 2012;7:e38636.

38. Henry JV, Winters N, Lakati A, et al. Enhancing the Supervision of Community Health Workers With WhatsApp Mobile Messaging: Qualitative Findings From 2 Low-Resource Settings in Kenya. Glob Health Sci Pract 2016;4:311-25.

39. Mayes T, De Freitas S. Technology-enhanced learning. In: Beetham H, Sharpe R. eds. Rethinking Pedagogy for a Digital Age: Designing for 21st Century Learning.Routledge London 2013:1730 .

40. Laurillard D, Oliver M, Wasson B, et al. Implementing technologyenhanced learning. In: Balacheff N, Ludvigsen S, de Jong T, eds. Technology-enhanced learning: Principles and products. Dordrecht: Springer, 2009.

41. Davies C, Eynon R. Education and Technology: Major Themes in Education: Routledge, 2016.

42. Selwyn N. Education and technology: Key issues and debates. London: Bloomsbury Publishing, 2016.

43. Sfard A. On Two Metaphors for Learning and the Dangers of Choosing Just One. Educational Researcher 1998;27:4-13.

44. Laurillard D. Pedagogical Forms of Mobile Learning: Framing Research Questions. Pachler N, ed. Mobile Learning: Towards a Research Agenda. London: UCL Institute of Education, 2007

45. Pachler N, Bachmair B, Cook J. Mobile Learning: Structures, Agency, Practices. New York: Springer, 2009.

46. Wali E, Winters N, Oliver M. Maintaining, changing and crossing contexts: an activity theoretic reinterpretation of mobile learning. Research in Learning Technology 2008;16:41-57.

47. Winters N. Mobile Learning in the Majority World: A Critique of the GSMA Position. In: Price S, Jewitt C, Brown B, eds. Sage Handbook of Researching Digital Technologies. London: Sage, 2013:402-11.

48. Mann KV. Theoretical perspectives in medical education: past experience and future possibilities. Med Educ 2011;45:60-8.

49. Winters N, O'Donovan J, Geniets A. A new era for community health in countries of low and middle income? Lancet Glob Health 2018;6:e489-e490.

50. Farmer P. Pathologies of Power: Health, Human Rights and the New War on the Poor. Berkeley: University of California Press, 2003. 